\title{
Estrategia conductiva del leño de Nothofagus alpina (Nothofagaceae), cuenca Lacar, Neuquén, Argentina
}

\author{
Wood conductive strategy of Nothofagus alpina (Nothofagaceae), \\ basin Lácar, Neuquén, Argentina
}

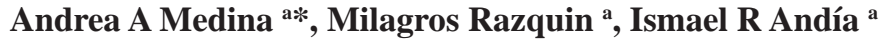 \\ *Autor de correspondencia: a Universidad Nacional del Comahue, Asentamiento Universitario San Martín de los Andes, \\ Pasaje de la Paz 235, San Martín de los Andes, 8370, Neuquén, Argentina, andrepampa@yahoo.com.ar
}

\begin{abstract}
SUMMARY
The hydraulic architecture of wood is one of the trees strategies of adaptations to their environment. The economic and ecological value of Raulí (Nothofagus alpina) in Argentina makes it necessary to generate knowledge that would contribute to an estimation of its adaptation capacity to variable growth conditions. The pattern of variation of conductive elements of the wood was determined and its anatomy was interpreted as adaptive strategy. Specimens of Lácar basin, Neuquén, Argentina, were studied; from them, slices were cut at $1.3 \mathrm{~m}$ height from the ground. The North orientated radius from each section was used for the extraction of samples every ten growth rings for the preparation of microscopic slides and macerations. Diameter and pore number per square millimeter, length of vessel elements and fibers, wall width and total diameter of fibers were measured, and net pore area, eco-anatomic vulnerability and pore grouping indexes were calculated. The major source of variation of the analyzed characteristics was the tree, related to age. Vulnerability (0.47) and pore grouping (1.71) indexes indicated a low vulnerability to cavitation. The efficiency in wood conduction significantly increased with age in detriment of conductive security. Conductive and mechanic resistance variables were shown to be highly correlated $\left(\mathrm{R}^{2}=0.71\right)$, suggesting connected functioning, adding conductive security to the wood.
\end{abstract}

Key words: conductive vulnerability, raulís wood, vessels grouping, cavitation.

\section{RESUMEN}

La arquitectura hidráulica del leño es una de las estrategias adaptativas de los árboles a su ambiente. El valor económico y ecológico del raulí (Nothofagus alpina) en Argentina hace necesario generar conocimiento que aporte a la estimación de su capacidad de adaptación a condiciones de crecimiento variables. Se determinó el patrón de variación de los elementos conductivos del leño y se realizó una interpretación de su anatomía como estrategia adaptativa. Se estudiaron ejemplares de la cuenca Lacar, Neuquén, Argentina, de los cuales se extrajo una rodela a 1,3 m de altura del suelo. Se utilizó el radio de orientación Norte de cada una de ellas para la extracción de probetas cada diez anillos de crecimiento a partir de las cuales se realizaron preparados microscópicos y macerados. Se midieron diámetro y número de poros por milímetro cuadrado, longitud de elementos de vaso y de fibras, espesor de pared y diámetro total de fibras y se calculó el área neta de poros e índices eco-anatómicos de vulnerabilidad y de agrupamiento de poros. La mayor fuente de variación de las características analizadas se presentó dentro del árbol en relación con la edad. Los índices de vulnerabilidad (0,47) y de agrupamiento de poros $(1,71)$ indicaron baja vulnerabilidad a la cavitación. La eficiencia en la conducción del leño aumentó significativamente con la edad en detrimento de la seguridad conductiva. Las variables conductivas y de resistencia mecánica se mostraron altamente correlacionadas $\left(\mathrm{R}^{2}=0,71\right)$, sugiriendo su funcionamiento en forma conectada, adicionando seguridad conductiva al leño.

Palabras clave: vulnerabilidad conductiva, madera de raulí, agrupamiento de poros, cavitación.

\section{INTRODUCCIÓN}

La arquitectura hidráulica de la madera de una especie puede ser entendida como una de las estrategias de adaptación al ambiente donde se desarrolla y por ello ser interpretada desde una perspectiva ecológica. Aspectos estructurales del tejido xilemático, como el diámetro, longitud, frecuencia y agrupamiento de sus células conductoras, permiten estimar el grado de seguridad o de eficiencia conductiva que presentan las especies en determinado sitio o etapa de su desarrollo.
La disposición de los poros en el tejido leñoso es consecuencia de dos fuerzas de selección contrapuestas: la eficiencia conductora del agua y la seguridad en la conducción de la misma (Moglia y López 2001a). Escasos poros de diámetros grandes contribuyen en mayor medida a la conductividad total que muchos de pequeño diámetro, ya que la capacidad conductiva del xilema se incrementa con la cuarta potencia del diámetro del poro. Por otro lado, poros pequeños, numerosos y agrupados tienden a garantizar la continuidad de la columna de agua en condiciones ambientales que predispongan a la cavitación como 
situaciones de estrés hídrico o térmico (Carlquist 1988, Lindorf 1994, Moglia y López 2001a). El resultado de la cavitación en el xilema es la aparición de embolismos u obstrucciones por gas en los vasos de conducción, lo cual reduce el transporte hidráulico del leño y puede resultar en un descenso de la conductancia estomática, una reducción fotosintética y el daño o muerte de ramas de la planta (Jacobsen et al. 2005).

Carlquist (1988) propuso el análisis de índices anatómicos, netamente eco-fisiológicos, como el de vulnerabilidad y el de agrupamiento de poros, para analizar la estrategia conductiva que presenta el leño de una determinada especie. El índice de vulnerabilidad relaciona el diámetro con el número de poros por unidad de superficie. Los valores más bajos arrojados por este índice indican una estrategia de seguridad conductiva del leño, con menor vulnerabilidad a la cavitación mientras que valores mayores señalan una estrategia de eficiencia en la conducción. El índice de agrupamientos de poros señala la capacidad del xilema para mantener la continuidad de la columna de agua ante la eventual obstrucción de algunos de ellos por embolias. Leños con valores más altos indican mayor agrupamiento de los elementos de vaso, señalando mayor seguridad conductiva.

Otra estrategia de seguridad conductiva puede presentarse en especies en las que el tejido de resistencia mecánica del leño está constituido por fibrotraqueidas, ya que estas células podrían realizar una acción subsidiaria y altamente segura de la conducción del agua en el leño en situaciones de estrés hídrico o térmico (Carlquist 1988, Moglia y López 2001a).

Por otro lado, la estructura del leño de una especie varía tanto entre individuos como dentro de ellos (Larson 1994), principalmente en relación con la edad. Respecto a las variables conductivas en general se reporta un aumento del diámetro, la longitud y el área neta de los elementos conductores con el incremento de la edad o diámetro de los árboles. La frecuencia de poros en el leño presenta un patrón de disminución de médula a corteza (Larson 1994, Giménez 2000, Moglia y López 2001b).

El raulí (Nothofagus alpina (Poepp. et Endl.) Oerst.= $N$. nervosa (Phil.) Dim. et Mil.) es un árbol deciduo de primera magnitud del Distrito Caducifolio de la Provincia Subantártica (Cabrera 1976). El área de su distribución natural en Argentina se reduce a la provincia de Neuquén, cubriendo 79.636 ha en una estrecha franja de unos $120 \mathrm{~km}$ de largo y 40 km como máximo de ancho, siguiendo los valles de lagos orientados de oeste a este (Sabatier et al. 2011). Es una importante especie forestal que ha sido aprovechada en forma histórica por las excelentes características estéticas y de trabajabilidad de su madera, utilizada para construcciones y carpintería en general (Tortorelli 1956). Su valor económico y ecológico en la región hace relevante la necesidad de generar conocimiento que aporte a la estimación de su capacidad de adaptación a condiciones de crecimiento variables, tanto entre sitios como frente al actual escenario de cambio climático.
Cabe destacar que la arquitectura hidráulica del leño es una entre varias estrategias adaptativas de las especies leñosas por lo que su estudio en forma combinada aportará mayor conocimiento sobre la capacidad de adaptación de $N$. alpina a diferentes condiciones de crecimiento.

Si bien existen en Argentina descripciones anatómicas del leño de N. alpina (Tortorelli 1956, Rivera 1988), no se cuenta hasta la actualidad con información sobre variabilidad de sus caracteres conductivos ni con interpretaciones ecológicas o adaptativas. Tampoco se ha comprobado aún la posible participación de las fibrotraqueidas, principales células de su tejido de resistencia mecánica (Tortorelli 1956, Rivera 1988), en la conducción del agua, lo que le adicionaría seguridad conductiva al leño.

Los objetivos de este trabajo son: 1) analizar la estrategia conductiva del leño de $N$. alpina a partir de interpretaciones de su anatomía, 2) comprobar la participación del tejido de resistencia mecánica del leño en la función conductiva y 3) determinar el patrón de variación de los elementos conductivos del leño dentro del árbol, en función de la edad, y entre árboles.

\section{MÉTODOS}

Los sitios de estudio se ubicaron en Quilanlahue y Nonthué (figura 1), cuenca Lacar, Distrito Caducifolio de la Provincia Subantártica (Cabrera 1976), también denominados Bosques Mesófilos de Patagonia por Roig (1998). Son áreas de bosque nativo mixto de N. alpina, N. obliqua y $N$. dombeyi y presentan similar índice de sitio (Attis Beltran 2010). El clima es templado húmedo con régimen pluviométrico mediterráneo. La precipitación media anual es 2.000 y $2.500 \mathrm{~mm}$, respectivamente, en Quilanlahue y Nonthue; la temperatura media anual es de $9^{\circ} \mathrm{C}$ y el régimen de humedad de los suelos es údico (Ferrer et al. 2006). Se estudiaron ocho ejemplares adultos (diámetro altura del pecho 40-60 cm), sanos, de estado sociológico dominante y buena forma de fuste de $N$. alpina.

De cada ejemplar se extrajo una rodela a $1,3 \mathrm{~m}$ de altura del fuste. Se utilizó el radio de orientación Norte de cada una de ellas para obtener probetas de leño destinadas a preparados microscópicos permanentes y macerados. Las probetas se extrajeron cada diez anillos de crecimiento desde la médula hacia la corteza (figura 2). La elección de este diseño de muestreo permitió analizar la variación del sistema de conducción con la edad de los árboles.

Siguiendo la metodología propuesta por el Comité de IAWA (1989) se analizaron como variables anatómicas: diámetro y número de poros por milímetro cuadrado, longitud de elementos de vaso, longitud, espesor de pared y diámetro total de fibras. Se calculó el área de poros multiplicando el valor del diámetro de poros por la frecuencia de los mismos por milímetro cuadrado (Sass y Eckstein 1995).

Se calcularon los siguientes índices (Carlquist 1988) como indicadores de la estrategia conductiva: 


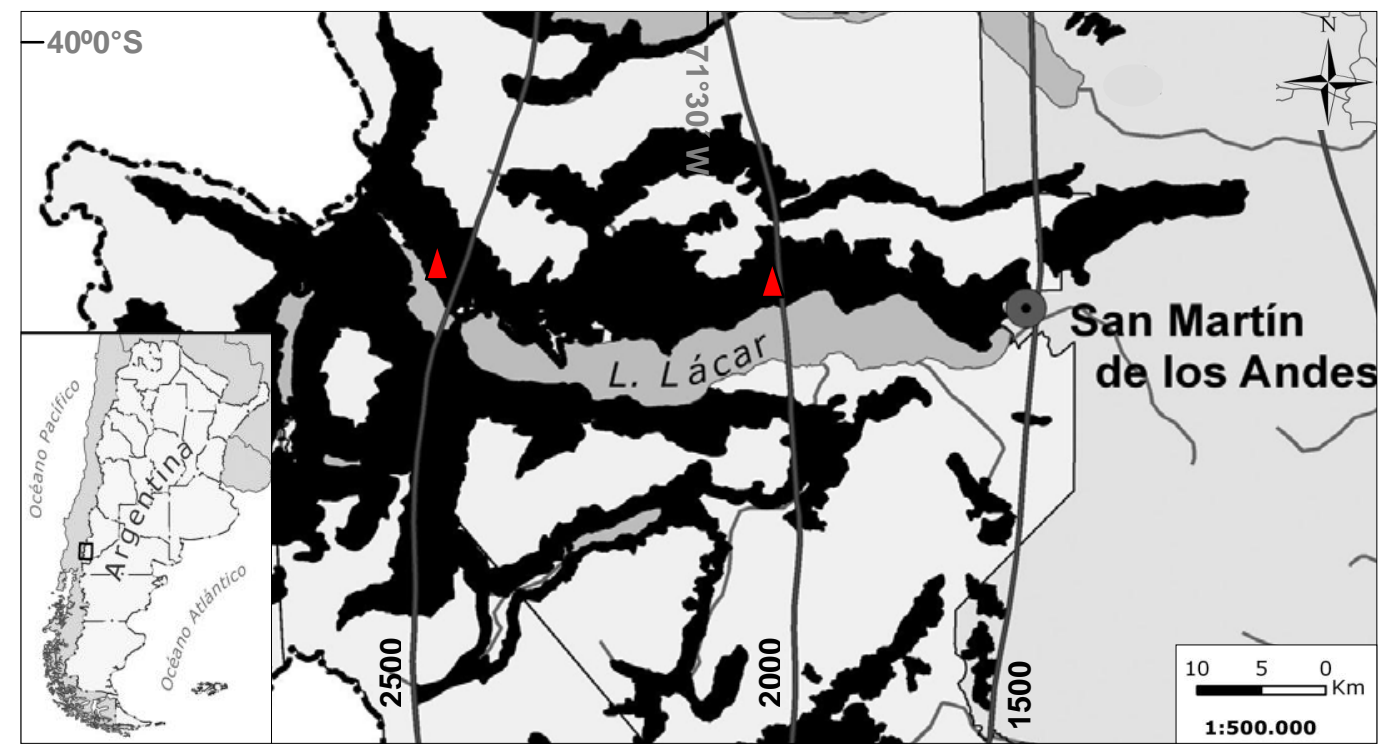

Figura 1. Mapa de distribución natural de Nothofagus alpina en Argentina (de Sabatier et al. 2011) con ubicación de los sitios de procedencia de los árboles analizados (Quilanlahue a la derecha; Nonthue a la izquierda).

Map of natural distribution of Nothofagus alpina in Argentina (from Sabatier et al. 2011) with site locations (Quilanlahue at rigth; Nonthue at left).

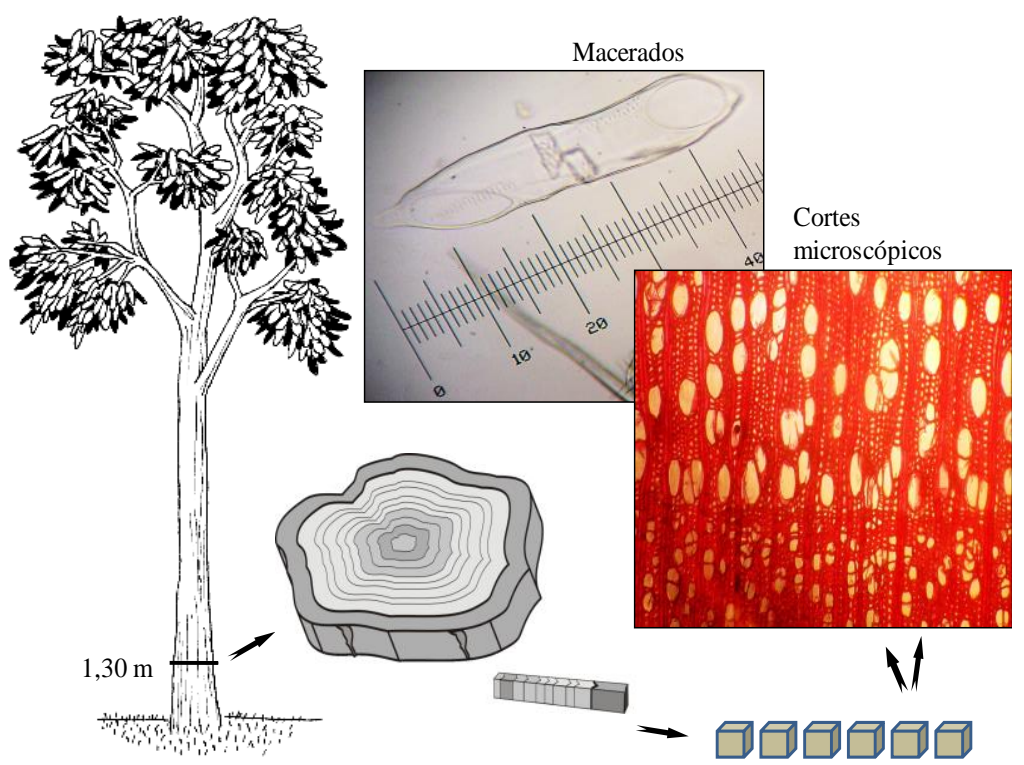

Figura 2. Esquema representando la toma de muestras en cada uno de los árboles analizados. Sampling methodology within every tree.

- Índice de vulnerabilidad (IV)= diámetros de poros $/ \mathrm{n}^{0}$ de poros $\mathrm{mm}^{-2}$. Valores menores indican mayor seguridad conductiva (menor vulnerabilidad a la cavitación).

- Índice de agrupamiento de poros (IAP) $=\mathrm{n}^{0}$ total de poros $/ \mathrm{n}^{\circ}$ de grupos de poros. Valores mayores indican mayor seguridad conductiva (menor vulnerabilidad en situaciones de estrés conductivo).

Se examinó la variación de estos índices y demás variables conductivas en relación con la edad de los árboles y entre los árboles. Se utilizó la prueba de Tukey para las comparaciones de medias y el nivel de significancia fue definido en todos los casos en $P<0,05$ (Devore 2005). Los análisis estadísticos se realizaron con el programa INFOSTAT (Di Rienzo et al. 2011).

Mediante análisis de correlación canónica se estudió la relación entre las variables asociadas al sistema conductivo y las variables que representan la resistencia mecánica del leño. Se seleccionaron las variables área de poros, 
longitud de elementos de vaso y diámetro de poros para expresar la función de conducción y longitud, diámetro y espesor de pared de fibras asociadas a la resistencia mecánica, con el supuesto de que ellas también intervienen en la conducción.

Las siguientes variables canónicas se generaron a partir de las variables escogidas para el análisis:

$\mathrm{C} 1=\mathrm{AP}+\mathrm{DP}+\mathrm{LV}$

$\mathrm{S} 1=\mathrm{LF}+\mathrm{EF}+\mathrm{DF}$

Donde, $\mathrm{AP}=$ área de poros; $\mathrm{DP}=$ diámetro de poros; $\mathrm{LV}=$ longitud de elementos de vaso; $\mathrm{LF}=$ longitud de fibras; $\mathrm{EF}=$ espesor de pared de fibras; $\mathrm{DF}=$ diámetro total de fibras.

Se obtuvo el coeficiente de correlación canónico y los coeficientes canónicos estructurales que relaciona ambas variables con el programa InfoStat (2011).

\section{RESULTADOS}

El leño de los ejemplares analizados presentó porosidad difusa con disminución gradual del diámetro de los elementos vasculares desde el inicio hacia la finalización del crecimiento anual. Los anillos de crecimiento estuvieron definidos por estrato de fibras con paredes engrosadas y aplanadas radialmente. Los elementos vasculares presentaron perforaciones simples terminales o laterales, raramente escalariformes, horizontales a oblicuos. Presentaron en general apéndices, de longitud variada, algunos alcanzando valores de hasta $300 \mu \mathrm{m}$ y puntuaciones vasculares opuestas, a veces escalariformes, con aréola de forma circular a hexagonal. La presencia de tílides en el interior de los elementos de vaso fue observada a partir de los 60 años de edad de los árboles. Se observaron escasas traqueidas con dos hileras de puntuaciones areoladas. El tejido de resistencia mecánica estuvo formado principalmente por fibrotraqueidas con abundantes puntuaciones areoladas con reborde reducido. Se observó baja frecuencia de fibras libriformes (con escasas puntuaciones simples) y de fibras septadas. En el cuadro 1 se expresaron los resultados estadísticos de los caracteres anatómicos analizados.

Los índices de vulnerabilidad y de agrupamiento de poros arrojaron valores medios que señalan una estrategia de seguridad conductiva del leño (cuadro 1).

El índice de vulnerabilidad aumentó de manera significativa con la edad (figura 4D), mientras que se registró una disminución del índice agrupamiento de poros con la edad de los árboles (figura 4E).

Los resultados del análisis de correlaciones canónicas indicaron que las variables que representan a la conducción se encuentran altamente relacionadas con las variables vinculadas con la resistencia mecánica del leño (cuadro 2).

Las variables diámetro de poros, número de poros por milímetro cuadrado, longitud de elementos de vaso, índice de vulnerabilidad e índice de agrupamiento de poros presentaron su mayor fuente de variación dentro del árbol, relacionada con las diferentes edades del leño, mientras que la variabilidad entre los árboles no fue significativa. $\mathrm{El}$ área de poros presentó variación significativa entre los árboles (figura 3) y no significativas entre las distintas edades analizadas.

El diámetro de poros y la longitud de los elementos vasculares aumentaron de manera significativa con la edad (figura 4A y $4 \mathrm{C}$ ) mientras que el número de poros $\mathrm{mm}^{-2}$ disminuyó significativamente de médula a corteza (figura 4B).

\section{DISCUSIÓN Y CONCLUSIONES}

El patrón de porosidad presente en el leño de $N$. alpina es similar al descrito en Fagus sylvatica L., Fagus orientalis Lipsky (Sass y Eckstein 1995, Pourtahmasi et al. 2011) y otros árboles del género Nothofagus (IAWA Committe 1989, Schweingruber 1993, Altube 2011). Estas especies se distribuyen también en áreas de climas templados con régimen pluviométrico mediterráneo, en los que las precipitaciones se

Cuadro 1. Valores medios, desviación estándar y coeficiente de variación de las variables medidas. Mean values, variation coefficient and standard deviation of measured variables.

\begin{tabular}{lccc}
\hline \multicolumn{1}{c}{ Característica } & $\begin{array}{c}\text { Promedio } \\
\text { general }\end{array}$ & $\begin{array}{c}\text { Desviación } \\
\text { estándar }\end{array}$ & $\begin{array}{c}\text { Coeficiente de } \\
\text { variación (\%) }\end{array}$ \\
\hline Diámetro de poros $(\mu \mathrm{m})$ & 46,60 & 16,13 & 34,62 \\
Número de poros $\mathrm{mm}^{-2}$ & 121,90 & 69,26 & 56,82 \\
Longitud de elementos de vasos $(\mu \mathrm{m})$ & 624,70 & 135,41 & 21,68 \\
Área de poros $\left(\mathrm{mm}^{2}\right)$ & 0,20 & 0,12 & 63,53 \\
Diámetro de fibrotraqueidas $(\mu \mathrm{m})$ & 15,23 & 5,38 & 35,36 \\
Espesor de pared de fibrotraqueidas $(\mu \mathrm{m})$ & 3,66 & 1,05 & 28,82 \\
Longitud de fibrotraqueidas $(\mu \mathrm{m})$ & $1.009,83$ & 207,33 & 20,53 \\
Índice de vulnerabilidad & 0,47 & 0,24 & 51,10 \\
Índice de agrupamiento de poros & 1,71 & 0,26 & 15,20 \\
\hline
\end{tabular}


Cuadro 2. Resumen de la correlación canónica y coeficientes canónicos estructurales. Conductive characters variation between ages and trees.

\begin{tabular}{|c|c|c|c|}
\hline \multicolumn{2}{|c|}{$\mathrm{R}^{2}$ Correlación canónica $=0,7064$} & Chi $^{2}(9)=45,213$ & $P=0,0000$ \\
\hline \multicolumn{4}{|c|}{ Coeficientes canónicos de la variable $\mathrm{C} 1$} \\
\hline & Correlación 1 & Correlación 2 & Correlación 3 \\
\hline Longitud de elementos de vaso & 0,52537 & $-0,81043$ & 0,96636 \\
\hline Diámetro de poros & 0,69912 & 0,90745 & $-1,22826$ \\
\hline Área de poros & $-0,39081$ & 0,32846 & 1,24586 \\
\hline \multicolumn{4}{|c|}{ Coeficientes canónicos de la variable S1 } \\
\hline & Correlación 1 & Correlación 2 & Correlación 3 \\
\hline Longitud de fibras & 0,83774 & $-0,40046$ & 0,50828 \\
\hline Diámetro total de fibras & 0,32930 & 0,94098 & $-0,21285$ \\
\hline Espesor de pared de fibras & $-0,14124$ & 0,23022 & 1,00365 \\
\hline
\end{tabular}

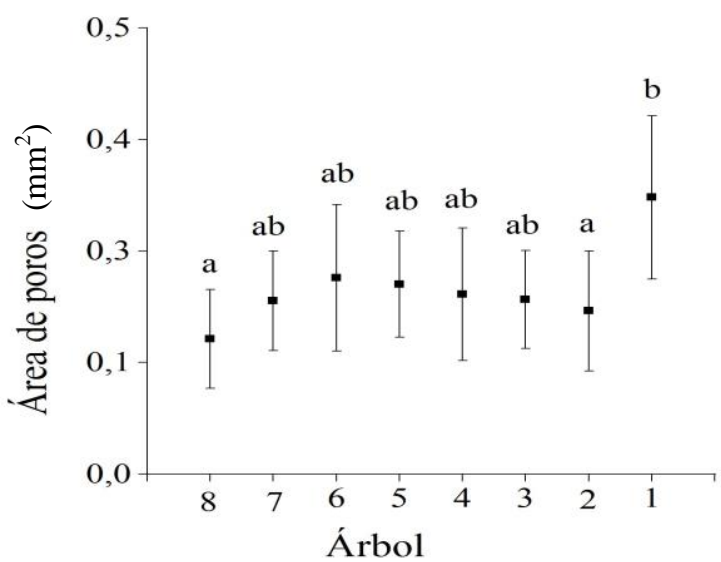

Figura 3. Área neta de poros $\mathrm{mm}^{-2}$ (promedios + desvío estándar) en cada uno de los árboles analizados. Valores promedios con letra distinta indican diferencias significativas entre edades (Tukey, $P<0,05$ ).

Variation of net vessel area by trees (means+ standard deviation). Averages values with different letters indicate significant differences among ages (Tukey, $P<0.05$ ).

concentran principalmente durante los meses de inactividad cambial de los árboles y los veranos son secos y calurosos.

De la descripción y mediciones anatómicas realizadas se observa un leño con características consideradas ventajosas para la conducción segura del agua en situaciones de estrés hídrico o térmico. Entre ellas, alta frecuencia de poros por unidad de superficie, poros agrupados y de tamaño reducido, elementos de vaso con placas de perforación simple, fibrotraqueidas como células principales del tejido de resistencia mecánica y presencia de traqueidas (Barajas-Morales 1985, Carlquist 1988, Lindorf 1994, Moglia y López 2001a) Dentro del área de distribución natural de $N$. alpina, los meses de actividad cambial de los árboles (octubre a marzo) coinciden con altas temperaturas, bajas humedades relativas del aire y fuertes y frecuentes vientos. Tales condiciones determinan valores de potencial hídrico atmosférico muy bajos y elevados flujos de transpiración en los árboles que predisponen a las columnas de agua intraxilemáticas a la cavitación. Por otro lado, la probabilidad de ocurrencia de heladas existe durante todo el año en la zona, las que pueden también desencadenar procesos de cavitación y embolismo en el xilema por congelación de la savia (Jacobsen 2005).

Los valores del índice de vulnerabilidad y del índice de agrupamiento de poros señalan una estrategia de marcada seguridad conductiva del leño de $N$. alpina. Valores menores a la unidad del índice de vulnerabilidad indican maderas con menor vulnerabilidad a la cavitación ya que presentan un leño con poros abundantes de diámetros pequeños capaces de garantizar la continuidad de la columna de agua en condiciones ambientales que predispongan a la cavitación. Respecto al índice de agrupamiento, mientras más se aleje su valor de la unidad (maderas con poros solitarios) mayor seguridad conductiva representa, indicando mayor agrupamiento de los poros en el leño y, consecuentemente, mayor capacidad del xilema para mantener la continuidad de la columna de agua ante la eventual obstrucción de algunos de ellos por embolias. Si bien no hay datos comparables de éstos índices para especies de bosques templados como $N$. alpina, si se reportan para especies de regiones semiáridas (Moglia y López 2001a), manglares (León 2001), bosques secos tropicales (León 2005) y diversos ambientes secos de Australia (Carlquist 1977).

La disminución de la seguridad y el aumento de la eficiencia en la conducción del leño con la edad de los árboles demuestran que las plantas más jóvenes seleccionan estrategias más seguras de conducción. La ganancia en eficiencia conductiva con la edad tiene seguramente relación con el mayor desarrollo general y radicular en particular de los árboles. Las fuerzas de selección entre la eficiencia y la seguridad en la conducción del agua en el xilema son contrapuestas (Moglia y López 2001a) por lo cual las plantas deben realizar una permanente "negociación” o "tradeoff' entre ellas.

Los valores arrojados por el análisis de correlaciones canónicas entre las variables conductivas y las variables 


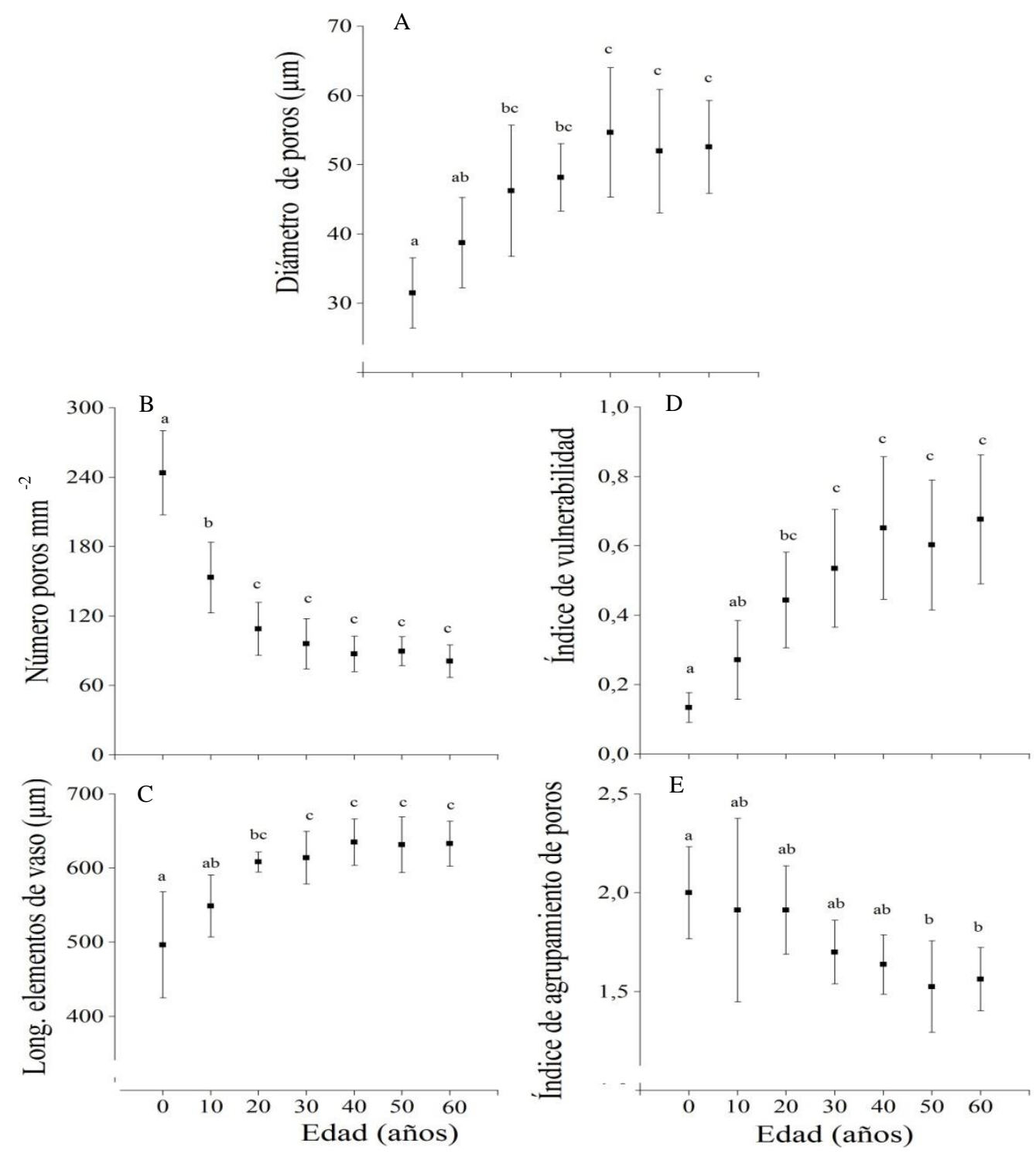

Figura 4. Variación en función de la edad de los árboles de: A) diámetro de poros; B) número de poros mm²; C) longitud de elementos de vaso; D) índice de vulnerabilidad y E) índice de agrupamiento de poros (promedios + desvío estándar). Valores promedios con letras distinta indican diferencias significativas entre edades (Tukey, $P<0,05$ ).

Variation by tree ages of A) vessel diameter, B) vessel number $\mathrm{mm}^{-2}$, C) vessel element length, D) vulnerability index and E) vessels grouping index (means + standard deviation). Averages values with different letters indicate significant differences among ages (Tukey, $P<0.05)$.

de resistencia mecánica analizadas muestran que las mismas se encuentran altamente correlacionadas, sugiriendo que funcionan en forma conectada, adicionando seguridad conductiva al leño de estos árboles. Esta estrategia de seguridad conductiva ha sido descrita en especies en las que el tejido de resistencia del leño está compuesto principalmente por fibrotraqueidas (Moglia y López 2001a). Si bien estas células cumplen funciones de resistencia mecánica, en situaciones de estrés hídrico o térmico pueden subsidiar la conducción, disminuyendo la vulnerabilidad conductiva del leño (Carlquist 1988).

La mayor variabilidad observada dentro del árbol y vinculada a las edades del leño en $N$. alpina coincide con el patrón descrito por Larson (1994), en el que se señala mayor variación de las características de la madera dentro del árbol, en relación principalmente con la edad, que entre árboles, aun creciendo estos en distintos sitios. Cabe resaltar que los árboles analizados en este trabajo proceden de lugares con similar índice de sitio (Attis Beltran 2010), lo que no permitió considerar efectos de sitio sobre las variables analizadas.

El aumento del diámetro y la longitud de los elementos conductores y la disminución de la frecuencia de poros de médula a corteza se reporta en especies de porosidad difusa (Larson 1994, Giménez 2000, Moglia y López 2001b). Estos patrones se relacionan tanto con la ganancia en efi- 
ciencia conductiva con la edad como con un proceso de maduración del leño.

El área de poros no presentó variación significativamente con la edad ya que es producto de una variable que aumenta (el diámetro de los poros) y otra que disminuye con la edad (el número de poros por milímetro cuadrado). $\mathrm{Su}$ variación significativa entre árboles indica diferencias en sus capacidades de transporte o vitalidad (Sap y Eckestein 1995) y podría ser utilizada como herramienta de selección de ejemplares en procesos de mejoramiento y domesticación forestal.

Los altos coeficientes de variación de las variables conductivas (cuadro 1) se atribuyen principalmente al diseño de muestreo utilizado, ya que se analizaron leños de distintas edades. Sin duda también existe una influencia de la variabilidad climática interanual sobre las características conductivas del xilema ya que los factores ambientales actúan como fuerzas modeladoras del número y tamaño de los elementos constitutivos del xilema (Carlquist 1988, Delgado et al. 2002).

Es importante tener en cuenta que la arquitectura hidráulica del leño es una entre varias estrategias de adaptación de las especies leñosas, como el tipo de estructura, suculencia y persistencia foliar, sistema radical, tasa de crecimiento y procesos fisiológicos especiales entre otras (Lindorf 1994). El estudio combinado de éstas características aportará a una interpretación más integral de la capacidad de adaptación de $N$. alpina a condiciones de crecimiento variables, tanto en escenarios de cambio climático como en proyectos de mejoramiento y domesticación forestal.

\section{AGRADECIMIENTOS}

A la Autoridad Interjuridiccional de las Cuencas de los ríos Neuquén, Limay y Negro (AIC), Secretaría Operativa y de Fiscalización (SOF), por el envío de datos climáticos de la zona de estudio. A Lic. Lorena Laffitte por su colaboración en los análisis estadísticos.

\section{REFERENCIAS}

Altube C, AA Medina, LN Laffitte. 2011. Aporte al conocimiento de la eco-anatomía de la madera de las especies del género Nothofagus de Neuquén, Argentina. Informe Práctica Laboral. Biblioteca del Asentamiento Universitario San Martín de los Andes, Neuquén, Argentina. Universidad Nacional del Comahue.19 p.

Attis Beltrán H. 2010. Análisis del crecimiento en altura dominante de Nothofagus nervosa y Nothofagus obliqua en la Cuenca de los lagos Lácar y Nonthué, Neuquén, Argentina. Informe de trabajo final de la carrera de Ingeniería Forestal, Facultad de Ciencias Agrarias y Forestales. Universidad Nacional de La Plata.

Barajas-Morales J. 1985 Wood structural differences between trees of two tropical forests in Mexico. IAWA Bulletin. New Series $N^{\circ}$ 8: 355-364.

Cabrera AL. 1976. Regiones fitogeográficas argentinas. Enciclo- pedia Argentina de Agricultura y Jardinería Tomo II Fascículo 1. Buenos Aires, Argentina. Editorial ACME. 85 p.

Carlquist S. 1977. Ecological factors in wood evolution: a floristic approach. American Journal of Botany 64(7): 887-896.

Carlquist S. 1988. Comparative wood anatomy. Berlín, Alemania. Springer Verlag. 460 p.

Delgado S, D Mendoza, R Villalba. 2002. Climatic influences on vessel size of Nothofagus pumilio in the southern Andes. Dendrochronology, environmental change and human history. 6th International Conference on Dendrochronology, Québec City, Canada.

Devore JL. 2005. Probabilidad y Estadística para Ingeniería y Ciencias. Sexta ed. Ed. Thomson. México D. F. 794 p.

Di Rienzo JA, F Casanoves, MG Balzarini, L Gonzales, M Tablada, CW Robledo. InfoStat versión 2011. Grupo InfoStat, FCA, Universidad Nacional de Córdoba, Argentina. URL http://www.infostat.com.ar

Ferrer J, JA Irisarri, JM Mendia. 2006. Suelos de la Provincia de Neuquén. Buenos Aires, Argentina. Instituto Nacional de Tecnología Agropecuaria. 226 p.

Giménez AM. 2000. Gradiente radial de los elementos anatómicos del leño en Schinopsis quebracho colorado (Schelcht.) Baril et. Meyer. Bosque 21(2): 37-45.

IAWA Committee (International Association of Wood Anatomists, NL). 1989. IAWA List of Microscopic features for hardwood identification. IAWA Bulletin 10 (3): 219-332. In Baas P, AJ Bolton, DM Catling eds. Wood Structure in Biological and technological research. Leiden Botanical Series No3. The Hague, The Netherland. Leiden Univ. Press. p. 157-181.

Jacobsen A, FW Ewers, R Brandon Pratt, WA Paddock III, SD Davis. 2005. Do xylem Fibers Affect Vessel Cavitation Resistance? Plant Physiology 139: 546-556.

Larson PR. 1994. The vascular cambium. Development and Structure. Springer Series in Wood Science. 720 p.

León HW. 2001. Anatomía del leño, aspectos ecológicos y filogenia en mangles de Venezuela. Revista Forestal Venezolana 45 (2): 191-203.

León HW. 2005. Anatomía ecológica del xilema secundario de un bosque seco tropical de Venezuela. Acta Botánica Venezolana 28(2): 257-274.

Lindorf H. 1994. Eco-anatomical wood features of species from a very dry tropical forest. IAWA Journal 15(4): 361- 376.

Moglia JG, CR López. 2001a. Estrategia adaptativa del leño Aspidosperma quebracho blanco. Madera y Bosques 7(2): 13-25.

Moglia JG, CR López. 2001b. Variabilidad radial de algunos caracteres anatómicos de Aspidosperma quebracho blanco. Bosque 22(2): 3-14.

Pourtahmasi K, N Lotfiomran, A Braüning, D Parsapajouh. 2011. Tree-ring width and vessel characteristics of Oriental Beech (Fagus orientalis) along an altitudinal gradient in the Caspian Forest, Northern Iran. IAWA Journal 32(4): 461-473.

Rivera SM. 1988. Revisión xilológica del género Nothofagus Bl. (Fagaceae) para la Argentina. Monografías Academia Nacional de Ciencias Exactas, Físicas y Naturales, Buenos Aires, Argentina (4): 73-84.

Roig FA. 1998. La Vegetación en la Patagonia. In Correa MN ed. Flora Patagónica: Parte I. Buenos Aires, Argentina: Colección Científica del INTA. p. 48-166,

Sabatier Y, MM Azpilicueta, P Marchelli, M González-Peñalba, L Lozano, L García, A Martínez, LA Gallo, F Umaña, D 
Bran, MJ Pastorino. 2011. Distribución natural de Nothofagus alpina y Nothofagus obliqua (Nothofagaceae) en Argentina, dos especies de primera importancia forestal de los bosques templados norpatagónicos. Boletín de la Sociedad Argentina de Botánica 46: 131-138.

Sass U, D Eckstein. 1995. The variability of vessel size in beech
(Fagus sylvatica L.) and its ecophysiological interpretation. Trees 9: 247-252.

Schweingruber F. 1993. Trees and Wood in Dendrochronology. Berlin, Germany. Springer Series in Wood Science. 402 p.

Tortorelli LA. 1956. Maderas y Bosques Argentinos. Buenos Aires, Argentina. Editorial ACME. 910 p.

Recibido: 17.05.12

Aceptado: 26.12.12 